\title{
Finite size effect on spread of resonance frequencies in arrays of coupled vortices
}

\author{
Andreas Vogel ${ }^{1}$, André Drews ${ }^{1,2}$, Mi-Young $\operatorname{Im}^{3}$, Peter Fischer ${ }^{3}$, and Guido Meier ${ }^{1}$ \\ ${ }^{1}$ Institut für Angewandte Physik und Zentrum für Mikrostrukturforschung, Universität Hamburg, 20355 Hamburg, Germany \\ ${ }^{2}$ Arbeitsbereich Technische Informatiksysteme, Universität Hamburg, 22527 Hamburg, Germany \\ ${ }^{3}$ Center for X-Ray Optics, Lawrence Berkeley National Lab, Berkeley, CA 94720, USA
}

\begin{abstract}
Dynamical properties of magnetic vortices in arrays of magnetostatically coupled ferromagnetic disks are studied by means of a broadband ferromagnetic-resonance (FMR) setup. Magnetic force microscopy and magnetic transmission soft X-ray microscopy are used to image the core polarizations and the chiralities which are both found to be randomly distributed. The resonance frequency of vortex-core motion strongly depends on the magnetostatic coupling between the disks. The parameter describing the relative broadening of the absorption peak observed in the FMR transmission spectra for a given normalized center-to-center distance between the elements is shown to depend on the size of the array.
\end{abstract}

Index Terms-Broadband ferromagnetic resonance measurements, Magnetic properties of nanostructures, Magnetization dynamics, Magnetostatic coupling.

\section{INTRODUCTION}

Small ferromagnetic structures, e.g., in the vortex state are of great scientific interest due to their dynamics on the subnanosecond timescale and potential technological applications as ultrafast and high-density digital storage devices [1]-[3]. In the last years, the dynamics of vortices have been studied intensely [4]-[7]. Vortices appear in thin-film disks when a curling magnetization pointing in-plane and along the edges is energetically favorable. The short-range exchange interaction forces the magnetization out-of-plane in the center of the disk. Vortex configurations are characterized by the vortex core polarization $p= \pm 1$ and the chirality $C= \pm 1$. The polarization indicates the out-of-plane magnetization direction of the core and the chirality indicates the in-plane curling direction of the magnetization around the core. Using a time-dependent external magnetic field, a gyration of the vortex core around its equilibrium position can be excited (gyrotropic mode). The sense of rotation only depends on the polarization $p$ following a right-hand rule.

For distances between neighboring structures which are smaller than their lateral size, the stray fields start to notably influence the dynamics [8]-[11]. We have recently shown that magnetostatic interaction leads to shifts of the eigenfrequency of the gyrotropic mode in $4 \mathrm{x} \mathrm{N}$ arrays of permalloy disks which can be detected as relative broadening of the absorption peak in broadband ferromagnetic-resonance (FMR) measurements [12]. The relative broadening is proportional to the inverse sixth power of the center-to-center distance $d$ normalized with the radium $\mathrm{R}$ of the disks. In the $4 \times \mathrm{N}$ arrays, the contributions to the broadening which are independent of the distribution of the core polarizations were found to be dominant. After applying an appropriate perpendicular bias field during the reset to the vortex configuration, the broadening was not significantly changed even though the polarization configuration is changed from random to a preferred direction. For a given ratio $d / \mathrm{R}$, the broadening depends on the size and the assembly of the array. Unequal numbers of next neighbors in the center in comparison to the border of the array significantly contribute to the experimentally observed relative broadening [12], [13].

Here, the distributions of the core polarizations and the chiralities within an array of permalloy disks are determined using magnetic force microscopy and transmission soft X-ray microscopy. We experimentally 
investigate the effect of the size of the array on the spread of resonance frequencies via broadband FMR measurements. The effective parameter analytically describing the relative broadening of the absorption peak observed in the FMR transmission spectra for a certain normalized center-to-center distance between the disks is shown to depend on the size of the array.

\section{ANALYTICAL DESCRIPTION}

Considering the contributions due to the magnetostatic interaction with the surrounding disks, the total energy of a vortex trapped in the disk $i$ within an array can be written as

$E_{i}\left(\overrightarrow{a_{i}}, \overrightarrow{a_{j}}\right)=E_{0}+\frac{\kappa}{2}\left|\overrightarrow{a_{i}}\right|+E_{r f}\left(\overrightarrow{a_{i}}\right)+\sum_{j \neq i} E_{\mathrm{int}}\left(\overrightarrow{a_{i}}, \overrightarrow{a_{j}}\right)$

$\mathrm{E}_{0}$ is the equilibrium energy $(|\mathrm{a}|=0)$, where $\mathrm{a}_{\mathrm{i}}=\mathrm{X}_{\mathrm{i}} / \mathrm{R}$ is the position of the $i$ th vortex core with respect to the center of the disk $i$. The second term represents the sum of the exchange energy and self-magnetostatic energy of a single off-centered vortex core, where the stiffness constant $k$ depends on the thickness $L$, the radius $R$, and the saturation magnetization $\mathrm{M}_{\mathrm{s}}$ of the disk [14]. The third term in (1) is the Zeeman energy in the radio-frequency magnetic field in x-direction around the central conductor of the coplanar waveguide which is used to excite the gyrotropic mode in the experiments. The fourth term is the sum over the energy contribution $E_{\text {int }}\left(a_{i}, a_{j}\right)$ due to magnetostatic interaction with the surrounding disks $j$ in the array

It is possible to numerically calculate the magnetostatic energy between the side surfaces of two neighboring disks $i$ and $j$ using the rigid vortex model [8]. An inverse sixth power dependence of the time-averaged magnetostatic energy on the normalized center-to-center distance can be found. Taking into account that the stray field of a ferromagnetic disk with an off- centered vortex core resembles to the field of a magnetic dipole located in the center of the disk, the energy contribution $E_{\text {int }}$ due to magnetostatic interaction can be expressed as [12]

$$
\mathrm{E}_{\text {int }}\left(\text { Error! Bookmark not defined. } \overrightarrow{a_{i}}, \overrightarrow{a_{j}}\right)=\frac{C_{i} C_{j}}{\left(d_{i j} / R\right)^{6}}\left(\eta_{i j x} a_{i x} a_{j x}-\eta_{i j y} a_{i y} a_{j y}\right) \text {. }
$$

The parameters $\eta_{i j x}$ and $\eta_{i j y}$ are a measure for the symmetry of the stray fields and depend on the orientation of the vector Error! Bookmark not defined.Error! Bookmark not defined. $\overrightarrow{d_{i j}}$ between the disks i and $j$. The time-dependent displacement of the vortex core in x-and y-direction depends on the chirality C. For a given direction of an external magnetic field, an opposite chirality leads to a vortex core displacement in the opposite direction. Since the positions $\overrightarrow{a_{i}}$ and $\overrightarrow{a_{j}}$ of the vortex cores depend on $\mathrm{C}_{\mathrm{i}}$ and $\mathrm{C}_{\mathrm{j}}$, respectively, the energy contribution $\mathrm{E}_{\mathrm{int}}\left(\overrightarrow{a_{i}}, \overrightarrow{a_{j}}\right)$ in $(2)$ finally does not depend on $\mathrm{C}_{\mathrm{i}}$ and $\mathrm{C}_{\mathrm{j}}$. The exciting radio-frequency field of the coplanar waveguide is linearly polarized (in x-direction) and thus only the displacement in xdirection is affected by the polarization. The stray field depends on the rotational direction of the vortex core given by the polarization. The stray field of the disk $j$ acts as additional magnetic field on the disk $i$. Induced dipoles obey an inverse sixth power dependence of 
The dynamics of the vortex core trapped in the $i$ th disk within the array can be described using the Thiele equation [15]:

$\overrightarrow{F_{i}}+\overrightarrow{G_{i}} \times \overrightarrow{v_{i}}+D \overrightarrow{v_{i}}=0$

Here, $\overrightarrow{F_{i}}=-\nabla E_{i}$ is the force acting on the core, Error! Bookmark not defined. $\overrightarrow{G_{i}}=-G_{o i} \overrightarrow{e_{z}}$ is the gyrovector,

$\overrightarrow{v_{i}}=R \cdot d \overrightarrow{a_{i}} / d t \quad$ is the velocity, and $\mathrm{D}$ is a damping parameter. Neglecting the damping $(\mathrm{D}=0)$, an expression for the approximated shift of the vortex eigenfrequency due to magnetostatic interaction between two disks $i$ and $j$ within an array can be obain from (2) and (3):

$$
\frac{f_{i}}{f_{0}}=1+\frac{1}{2 \kappa}\left(\frac{d_{i j}}{R}\right)^{-6}\left(p_{i} p_{j} \eta_{i j x}-\eta_{i j y}\right) .
$$

Note that a part of the frequency shift in (4) is independent of the polarization configuration and only depends on the normalized center-to-center distance $d_{i j} / R$ and the orientation of the vector between disk $i$ and $j$. The total frequency shift due to magnetostatic interaction with all surrounding disks in an array with constant center-to-center distances along the axis can be described via an effective parameter $\eta_{i}{ }^{\prime}$ varies depending on the distribution of the core polarizations as well as on the size and the assembly of the array. Unequal numbers of next neighbors in the center in comparison to the border of the array lead to a change of the effect parameter $\eta_{i}{ }^{\prime}$. Considering the different elements within an array, this leads to a spread of resonance frequencies which is proportional to the inverse sixth power of the normalized center-to-center distance:

$$
\frac{\Delta f}{f_{0}}=\Delta \eta^{\prime}\left(\frac{d}{R}\right)^{-6}
$$

By varying the normalized center-to-center distance in $4 \times \mathrm{N}$ arrays of permalloy disks, we have experimentally confirmed the inverse sixth power dependence of the broadening $\Delta \mathrm{f}$ in FMR measurements [12]. In the following, we vary the size of the array for a given ration $d / R$ to investigate the influence on the spread of the effective parameter $\Delta \eta^{\prime}$.

\section{SAMPLE PREPARATION AND EXPERIMENTAL METHODS}

Various arrays with dimensions between $4 \times 300$ and $2 \times 2$ ferromagnetic disks with a radius of $R=500 \mathrm{~nm}$ are fabricated using electron-beam lithography and lift-off processing. Polycrystalline permalloy $\left(\mathrm{Ni}_{80} \mathrm{Fe}_{20}\right)$ with a thickness of $L=50 \mathrm{~nm}$ is thermally evaporated onto $100 \mathrm{~nm}$ thin silicon-nitride membranes for X-ray measurements and gallium arsenide substrates as well as silicon substrates with a $300 \mathrm{~nm}$ silicon oxide coating for electrical measurements. To perform electrical measurements, a coplanar waveguide is deposited on top of the disks via dc magnetron sputtering of $125 \mathrm{~nm}$ gold. To optimize the signal-to-noise ration, the signal line splits into four $800 \mathrm{~nm}$ wide lines which are each aligned with the centers of the disks. In the case of smaller arrays, several arrays are placed along the signal line separated by a distance which prevents coupling. The normalized center-to-center distance is measured using scanning electron microscopy and ranges from $d / R=2.15$ to $d / R=2.36$ between the elements along the axis. 
The polarizations $p$ of the magnetic vortex cores trapped in the center of the disks are resolved using magnetic force microscopy. Furthermore, transmission soft X-ray microscopy is used to determine the distribution of the chiralities C. The X-ray measurements have been performed at beamline 6.1.2 of the Advanced Light Source in Berkeley, CA, USA. Magnetic contrast is provided via the X-ray magnetic circular dichroism (XMCD) at the $\mathrm{Ni}_{3}$-absorption edge [16]. The microscope permits a spatial resolution down to $15 \mathrm{~nm}$ using Fresnel-zone plates [17]. The synchrotron delivers a constant electron-beam current of $500 \mathrm{~mA}$ for stable and intense Xray illumination. Samples are illuminated by circularly polarized X-rays with an incident energy of $852.7 \mathrm{eV}\left(\mathrm{Ni} \mathrm{L}_{3}\right.$-absorption edge). Magnetic imaging is realized in an in-plane geometry where the sample surface is mounted under an angle of $60^{\circ}$ with respect to the beam direction. Thus, a contrast due to the in-plane magnetization is detected.

Transmission spectra are measured at room temperature by means of a broadband ferromagnetic resonance setup using a vector-network analyzer [18]. The coplanar waveguide is contacted via GSG (Ground-Signal-Ground) probes. A sinusoidal signal of $-8 \mathrm{dBm}$ is driven through the waveguide which leads to a radio-frequency magnetic field of up to 0.3 mTacting in the plane of the disks. A static external bias field $\overrightarrow{H_{0}}$ Can be applied parallel to the waveguide. Prior to each frequency sweep, the disks are reset via alternating reduction of the bias field starting from saturation.

\section{EXPERIMENTAL RESULTS AND DISCUSSION}

$<$ FIG. $1>$

The superimposed atomic force and magnetic force micrographs shown in Fig. 1(a) reveal that the polarizations $p$ are randomly distributed in the investigated arrays. Figures 1(b) and 1(c) show differential X-ray images which are obtained by comparison of the magnetization configuration at zero field with the magnetization configuration at saturation by an inplane magnetic field. It is confirmed that the chiralities for a normalized center-to-center distance $d / R=2.2$ are randomly distributed as well and not influenced by magnetostatic interaction during the nucleation of the vortex state.

$<$ FIG. $2>$

Typical FMR transmission spectra are shown in Fig. 2. The transmission signal is reduced [dark color in Fig. 2(a)] when energy is absorbed in the gyrotropic mode [5]. An in-plane bias field $\overrightarrow{H_{0}}$ leads to a translation of the equilibrium position of the vortex core. The shift of the absorption peak in Fig. 2(a) depending on the magnetic field $\overrightarrow{H_{0}}$ can be explained by considering anharmonic contributions to the magnetic potential of the disk. The relative broadening $\left(\Delta f-\Delta f_{\min }\right) / f_{0}$ is observed in all arrays, see Figs. 2(b) and 2(c). Note that arrays on both substrates show comparable results (not shown).

For a single disk, the linewidth of the vortex resonance at zero field depends on the eigenfrequency, the Gilbert damping parameter, the thickness dependent vortex-core radius, and the radius $R$ of the disk [20]. The experimentally observed eigenfrequency $f_{0}$ of elements with nominally equal shape often varies in the order of half of the linewidth of the vortex resonance [21]. A minimum linewidth Error! Bookmark not defined. $\Delta f_{\min }$ of the FMR spectrum arises from the superposition of resonances of the single elements of the arrays of disks and is thus subtracted from the experimentally observed broadening before comparison 
to the analytical description. Figure 2(d) shows the FMR spectrum of a $4 \times 180$ array with a normalized center-to-center distance $d / R$ $=4.12$, where the coupling between the disks is mostly prevented.

$<$ FIG. $3>$

The experimental determination of the relative broadening in the FMR spectra at zero bias field for different arrays enable to calculate the spread $\Delta \eta^{\prime}$ of the effective parameter $\eta_{i}$ ' using (6). The normalized center-to-center distance $d / R$ has been measured for each array via scanning electron microscopy. Figure 3 shows the mean value of $\Delta \eta$ ' as a function of the size of the array. Only a small difference between the $4 \times 300$ and the $4 \times 4$ arrays has been observed which indicates that a further increase of the size of the array in $y$-direction (from $4 \times 4$ to $4 \times 300$ ) does not significantly change the spread of resonance frequencies of the elements. But for arrays of $3 \times 3$ and $2 \times 2$ disks, the FMR spectra reveal a strong decrease of $\Delta \eta$ '. Less neighbors interact in small arrays which could yield a smaller effective parameter $\eta_{i}$ ' for a disk $i$. The number of disks which are surrounded by other disks decreases in comparison to disks which are at the border of an array. In a $3 \times 3$ array, there are disks with two, three and eight next neighbors whereas in a $2 \times 2$ array, each disk has only two next neighbors.

\section{CONCLUSION}

Magnetic force microscopy and magnetic transmission soft X-ray microscopy have been used to investigate the distribution of the core polarizations and the chiralities in arrays of magnetostatically coupled permalloy disk. For a normalized center-to-center distance $d / R=2.2$ both distributions are shown to be still random and thus not influenced by magnetostatic interaction during the vortex nucleation. FMR transmission spectra of arrays $(2.15 \leq d / R \leq$ 2.36) of different size $(2 \times 2$ to $4 \times 300)$ reveal a relative broadening of the absorption peak due to a spread of resonance frequencies of the gyrotropic mode. The effective parameter describing the relative broadening for a certain normalized center-tocenter distance between the disks depends on the size of the array. For smaller arrays, the spread of resonance frequencies due to magnetostatic interaction has been shown to decrease.

\section{ACKNOWLEDGMENT}

We thank U. Merkt for continuous support and M. Volkmann for superb technical assistance. Financial support of the Deutsche Forschungsgemeinschaft via the Sonderforschungsbereich 668 is gratefully acknowledged. Operation of the X-ray microscope is supported by the DOE, Office of Science, under contract DE-AC02-05-CH11231.

\section{REFERENCES}

[1] B. N. Engel, J. Åkerman, B. Butcher, R. W. Dave, M. DeHerrera, M. Durlam, G. Grynkewich, J. Janesky, S. V. Pietambaram, N. D. Rizzo, J. 
M. Slaughter, K. Smith, J. J. Sun, and S. Tehrani, “A 4-Mb toggle MRAM based on a novel bit and switching method", IEEE Trans. Magn., vol. 41, pp. 132-136, Jan. 2005.

[2] D. A. Allwood, Gang Xiong, M. D. Cooke, C. C. Faulkner, D. Atkinson, N. Vernier, and R. P. Cowburn, "Submicrometer Ferromagnetic NOT Gate and Shift Register", Science, vol. 296, pp. 2003-2006, Jun. 2002.

[3] S. Bohlens, B. Krüger, A. Drews, M. Bolte, G. Meier, and D. Pfannkuche, "Current controlled random-access memory based on magnetic vortex handedness", Appl. Phys. Lett., vol. 93, pp. 142508-1142508-4, Oct. 2008.

[4] M. Bolte, G. Meier, B. Krüger, A. Drews, R. Eiselt, L. Bocklage, S.

Bohlens, T. Tyliszczak, A. Vansteenkiste, B. VanWaeyenberge, K. W. Chou, A. Puzic, and H. Stoll, "Time-Resolved X-Ray Microscopy of Spin-TorqueInduced Magnetic Vortex Gyration”, Phys. Rev. Lett., vol.

100, pp. 176601-1-176601-4, Apr. 2008.

[5] K. S. Buchanan, P. E. Roy, M. Grimsditch, F. Y. Fradin, K. Yu.

Guslienko, S. D. Bader, and V. Novosad, "Soliton-pair dynamics in patterned ferromagnetic ellipses", Nat. Phys., vol. 1, pp. 172-176, Dec. 2005 .

[6] S.-K. Kim, K.-S. Lee, Y.-S. Yu, and Y.-S. Choi, "Reliable low-power control of ultrafast vortex-core switching with the selectivity in an array of vortex states by in-plane circular-rotational magnetic fields and spin- polarized currents", Appl. Phys. Lett., vol. 92, pp. 022509-1-022509-3, Jan. 2008.

[7] R. Antos, Y. Otani, and J. Shibata, "Magnetic Vortex Dynamics“, J. Phys. Soc. Jpn., vol. 77, pp. 031004-1-031004-8, Mar. 2008.

[8] J. Shibata, K. Shigeto, and Y. Otani, "Dynamics of magnetostatically coupled vortices in magnetic nanodisks", Phys. Rev. B, vol. 67, pp. 224404-1-224404-4, Jun. 2003.

[9] K. L. Metlov, "Quasiuniform In-Plane Magnetization State of Thin Cylindrical Dots in a Square Array and Related Anisotropy", Phys. Rev. Lett., vol. 97, pp. 127205-1-127205-4, Sept. 2006.

[10] T. Kimura, Y. Otani, H. Masaki, T. Ishida, R. Antos, and J. Shibata, "Vortex motion in chilarity-controlled pair of magnetic disks", Appl. Phys. Lett., vol. 90, 132501-1-132501-3, Mar. 2007.

[11] Y. Liu, Z. Hou, S. Gliga, and R. Hertel, "Influence of the dynamic dipolar interaction on the current-induced core switch in vortex pairs", Phys. Rev. B, vol. 79, pp. 104435-1-104435-4, Mar. 2009.

[12] A. Vogel, A. Drews, T. Kamionka, M. Bolte, and G. Meier, "Influence of Dipolar Interaction on Vortex Dynamics in Arrays of Ferromagnetic Disks", Phys. Rev. Lett., vol. 105, pp. 037201-1-037201-4, Jul. 2010.

[13] V. Novosad, Materials Science Division and Center for Nanoscale Materials, Argonne National Laboratory, Argonne, IL 60439, USA, private communication, 2010 .

[14] K. Yu. Guslienko, V. Novosad, Y. Otani, H. Shima, and K. Fukamichi, "Magnetization reversal due to vortex nucleation, displacement, and annihilation in submicron ferromagnetic dot arrays", Phys. Rev. B, vol. 65, pp. 024414-1-024414-4, Dec. 2001.

[15] A. A. Thiele, "Steady-State Motion of Magnetic Domains", Phys. Rev. Lett., vol. 30, pp. 230-233, Feb. 1973.

[16] C. T. Chen, F. Sette, Y. Ma, and S. Modesti, "Soft-x-ray magnetic circular dichroism at the $L_{2,3}$ edges of nickel", Phys. Rev. B, vol. 42, pp. 7262-7265, Oct. 1990.

[17] W. Chao, B. H. Harteneck, J. A. Liddle, E. H. Anderson, and D. T. Attwood, "Soft X-ray microscopy at a spatial resolution better than $15 \mathrm{~nm}$ ", Nature (London), vol. 435, pp. 1210-1213, Jun. 2005.

[18] J. Podbielski, F. Giesen, and D. Grundler, „Spin-Wave Interference in Microscopic Rings”, Phys. Rev. Lett., vol. 96, pp. 167207-1-167207-4, Apr. 2006.

[19] I. Horcas, R. Fernandez, J.M. Gomez-Rodriguez, J. Colchero, J. Gomez- Herrero and A. M. Baro, "WSXM: A software for scanning probe microscopy and a tool for nanotechnology", Rev. Sci. Instrum., vol. 78, pp. 013705-1-013705-8, Jan. 2007.

[20] K. Yu. Guslienko, "Low-frequency vortex dynamic susceptibility and relaxation in mesoscopic ferromagnetic dots", Appl. Phys. Lett., vol. 89, pp. 022510-1022510-3, Jul. 2006.

[21] T. Kamionka, Institut für Angewandte Physik und Zentrum für Mikrostrukturforschung, Universität Hamburg, 20355 Hamburg, Germany, private communication, 2009. 


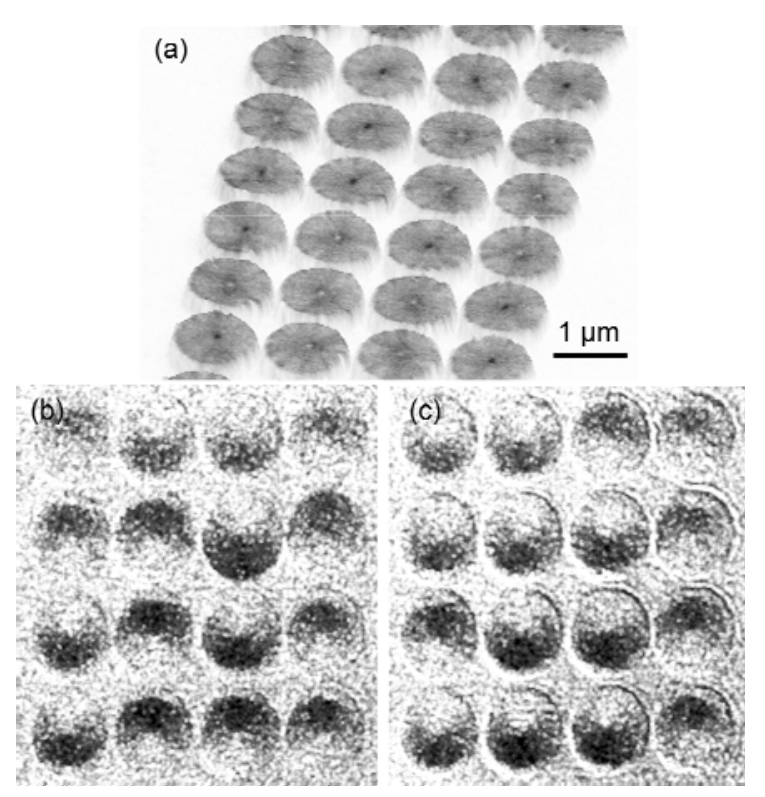

FIG. 1 (a) Superimposed atomic force and magnetic force micrographs [19] of an array of permalloy disks with a radius of $R=500 \mathrm{~nm}$ and a center-to-center distance of $d=1.1 \mu \mathrm{m}$. Differential MTXM images at zero field with respect to the saturated state are shown for two different positions (b) and (c) of a sample with arrays of $4 \times 4$ disks.
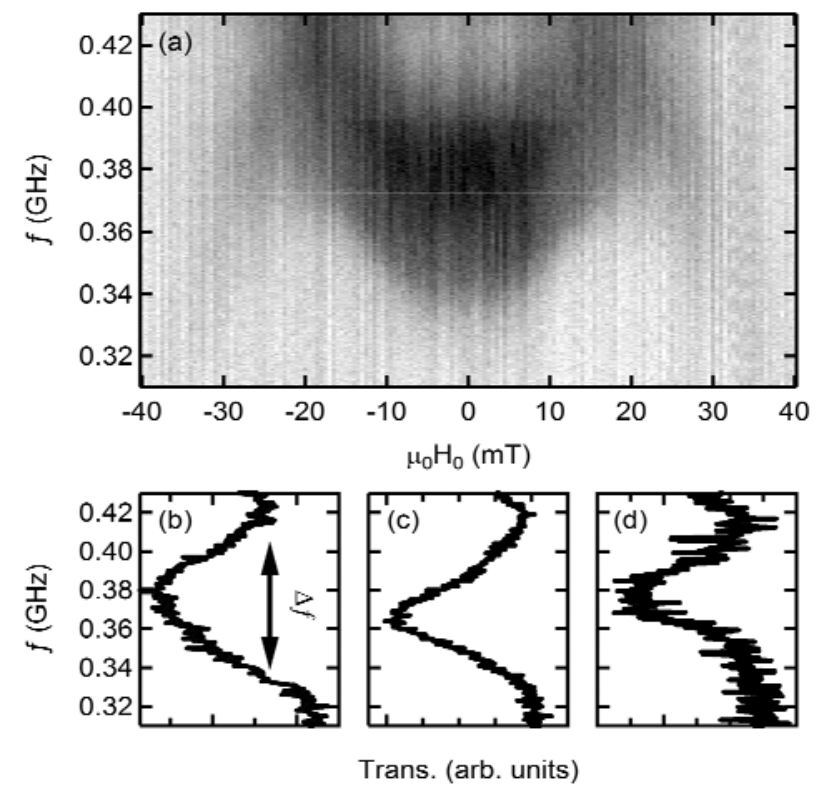

FIG. 2 (a) FMR transmission spectra of a $4 \times 300$ array of permalloy disks with a normalized center-to-center distance of $d / R=2.36$. An external bias field parallel to the waveguide is successively increased from $\mu_{0} H_{0}=-40 \mathrm{mT}$ to $40 \mathrm{mT}$. The dark color corresponds to reduced transmission. (b) Spectrum at zero bias field for the array shown in (a). (c) Spectrum at zero bias field for

$2 \times 2$ arrays with $d / R=2.32$. (d) Spectrum at zero bias field for a $4 \times 180$ array with $d / R=4.12$. 


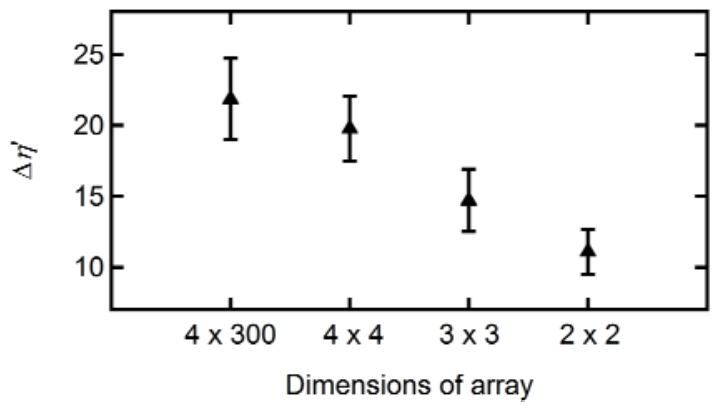

FIG. 3 Parameter $\Delta \eta^{\prime}$ describing the relative broadening of the absorption peak in the FMR transmission spectra at zero bias field for a given normalized center-to-center distance $d / R$ versus the size of the arrays. Black triangles depict mean values of the experimental data. 


\section{DISCLAIMER}

This document was prepared as an account of work sponsored by the United States Government. While this document is believed to contain correct information, neither the United States Government nor any agency thereof, nor the Regents of the University of California, nor any of their employees, makes any warranty, express or implied, or assumes any legal responsibility for the accuracy, completeness, or usefulness of any information, apparatus, product, or process disclosed, or represents that its use would not infringe privately owned rights. Reference herein to any specific commercial product, process, or service by its trade name, trademark, manufacturer, or otherwise, does not necessarily constitute or imply its endorsement, recommendation, or favoring by the United States Government or any agency thereof, or the Regents of the University of California. The views and opinions of authors expressed herein do not necessarily state or reflect those of the United States Government or any agency thereof or the Regents of the University of California. 